\title{
Research on Temperature Control System of Plant Factory Based on Particle Swarm Optimization
}

\author{
Shigang Cui , Jiejie Chen ${ }^{+}$, Xingli Wu , Lin He, Yongli Zhang \\ Tianjin University of Technology and Education, Tianjin Key Laboratory of Information Sensing \& \\ Intelligent Control, Tianjin, China
}

\begin{abstract}
In order to solve the problems of complex temperature control and inaccurate regulation in plant factories, this paper starts from the actual model of plant factory, uses STM32 processor with embedded Cortex-M3 as the core, and collects the plant factory through Modbus fieldbus technology. Temperature information, using the MATLAB tool to derive the temperature control model of the plant growth cabinet. Finally, the fuzzy control algorithm and particle swarm optimization (PSO) optimization algorithm are used to control the temperature control model with two different PIDs. By establishing the MATLAB simulation model, the optimal control method under the plant growth cabinet model is obtained. The results show that compared with fuzzy PID, PID control based on PSO algorithm has good stability and regulation, and plants based on this environment can get better growth.
\end{abstract}

Keywords: plant growth cabinet, temperature control, modbus fieldbus, PSO algorithm, fuzzy PID control

\section{Introduction}

The plant growth cabinet is essentially a micro-plant factory that achieves continuous production by controlling the environment within the facility [1-2]. Temperature belongs to one of the basic elements in the plant factory control system. At present, China has extensive research on temperature control, and the temperature control system has also been applied to various fields. The greenhouse system is a complex system. Due to the large coupling in the greenhouse system, it is difficult to achieve optimal control if traditional control methods are used. Proportional-integral-derivative (referred to as PID) control algorithm is one of the classic algorithms in control systems. Domestic and foreign scholars have also done a lot of research on PID algorithm. However, for plant growth cabinets (greenhouse systems), traditional PID control does not have the ability to be intelligent, self-optimizing and adaptive. In this paper, the particle swarm optimization (PSO) algorithm is used to optimize the PID parameters, and the optimization factor $k p, k i, k d$ in the controller is optimized. The shortcomings of traditional PID parameter tuning depend on experience are solved, and the PID parameter adjustment is more intelligent [3]. At the same time, the fuzzy PID control is compared. Compared with the fuzzy control, the particle swarm optimization PID is more suitable for the control of the plant growth cabinet temperature control model.

\section{Temperature Control Modeling}

Referring to the basic method of modeling mathematical model, there are two main methods for the establishment of temperature control model for this environment: mechanism method and experimental method. When the model is established by the mechanism method, it is necessary to consider not only the heat exchange between the temperature control system and the indoor air in the plant growth cabinet, but also the interaction between the indoor and outdoor air convection heat, and the plant transpiration. Some of the physical quantities to be tested have problems of measurement process complexity and measurement

\footnotetext{
+ Corresponding author. Tel.: 18526879830

E-mail address: cjj462880005@qq.com.
} 
error, which cannot fully reflect the actual situation of the system. Therefore, the system uses experimental methods for mathematical modeling. Using the experimental method for mathematical modeling can not only truly reflect the actual temperature changes, but also effectively avoid some coupling relationships in the temperature control system. By applying a step signal to the controlled object of the system at a certain point in the actual process, the current temperature value is recorded. By consulting the relevant literature, it can be seen that most of the temperature systems can be approximated by a first-order inertial link plus a first-order pure lag link, therefore the transfer function of the system can assume the formula (1).

$$
G(S)=\frac{K^{*} e^{-\tau s}}{T s+1}
$$

where $K, T, \tau$ represents the static gain, inertia time constant, and lag time of the system. Using the MATLAB system identification tool, as shown in Figure 1, the system transfer function of the controlled system can be obtained as the formula (2).

$$
G(S)=\frac{16.191 * e^{-29.12 s}}{849.81 s+1}
$$

This function is the basis of the system mathematical model, and the matching degree of the function with the step response curve is $95.81 \%$, as shown in Fig. 2, which is consistent with the response curve, which can well reflect the transmission characteristics of the system.

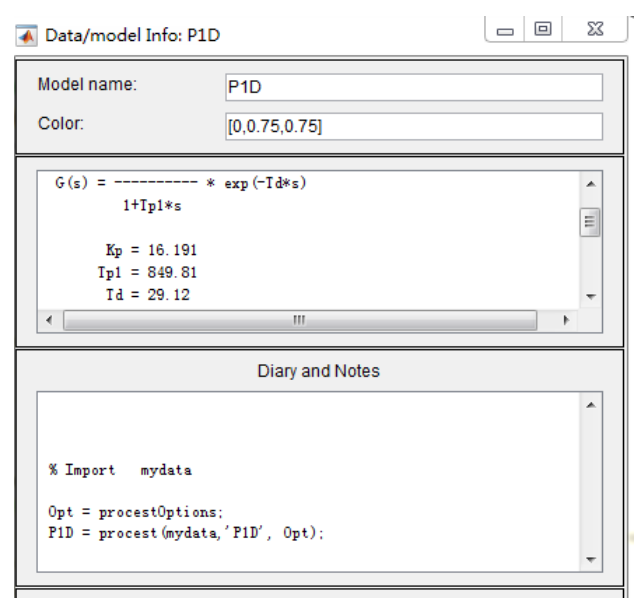

Fig. 1: Generate transfer function

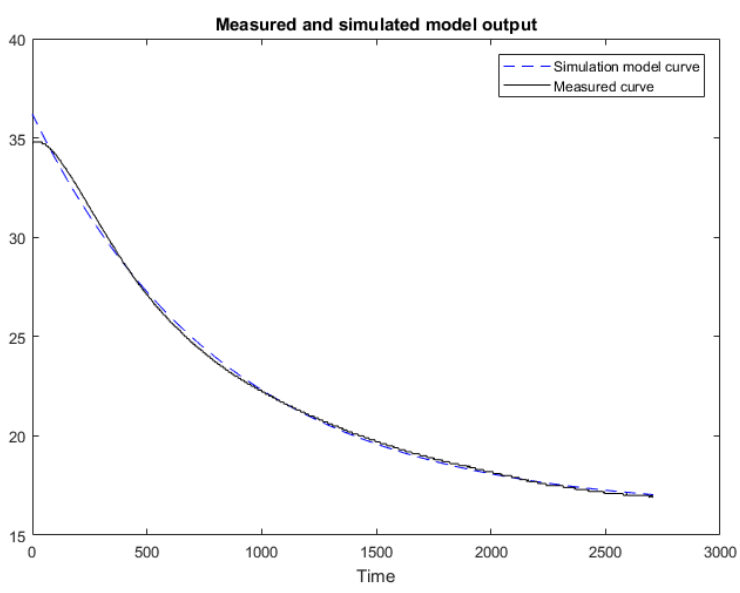

Fig. 2: Transfer function matching curve caption

\section{Fieldbus}

The plant growth cabinet is a control system composed of a plurality of subsystems, wherein the temperature control system is one of the important systems, and is composed of an STM32 processor, a sensor system and an execution unit, and the three are connected by Modbus, which not only solves the tradition. The redundancy of the wiring also ensures the stability of the data transmission. In order to ensure the accuracy of the collected temperature data, the system uses multiple sensors to collect at multiple locations and process them to obtain idealized data. The block diagram of its temperature control system is shown in Figure 3. The Modbus protocol is shown in Table 1.

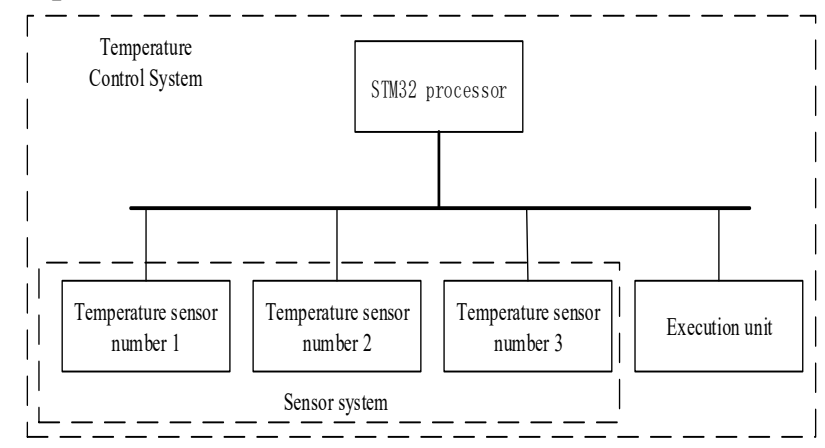

Fig. 3: The system Block Diagram 
Table 1: Data protocol table

\begin{tabular}{c|c}
\hline \hline Bits & Data \\
\hline 0 & Address \\
\hline 1 & Function instruction code \\
\hline 2 & Register data \\
\hline 3 & Register data \\
\hline 4 & Register data \\
\hline 5 & Register data \\
\hline 6 & CRC data \\
\hline 7 & CRC data \\
\hline \hline
\end{tabular}

\section{Fuzzy PID}

\subsection{Input and output variables and domains}

In some complex control fields, the problem of not being able to accurately build mathematical models can be solved by fuzzy control. The fuzzy control system is generally composed of four parts: fuzzy controller, input/output interface device, generalized object and sensor [4]. In fuzzy systems, design and adjustment controllers play an important role in fuzzy control systems. The design of the fuzzy controller is mainly composed of the following aspects.

The dimension of the fuzzy controller represents the accuracy of the control, and the number of input variables of the fuzzy controller determines the dimension of the fuzzy control [5]. The input variables of one-dimensional fuzzy control usually only select the error as the input quantity, but there is a problem that the dynamic control performance is not good. The three-dimensional fuzzy control has high dimensionality and finer control, but the control rules are too complicated and the control algorithm is difficult to implement. Therefore, two-dimensional fuzzy controllers are widely selected at present, and the two-dimensional controller model is shown in Fig. 4. In this study, two input variables were used, and the three output variables were used to control the temperature control model, the input variable deviation $c 1=2, c 2=2$, and the deviation change rate $e c$, and the output variable are $k p, k i, k d$. After many experiments, the $e, e c$ domain is finally set to [-2, 2], The domain of $k p, k i, k d$ is set to [-0.5, 0.5], [-0.1, 0.1], [-0.1, 0.1]. After completing the domain setting, it is necessary to quantify the input and output. The determination of the quantization factor is more important, which not only affects the dynamic quality of the system, but also affects the stability of the system. The quantization factor is expressed in accordance with the formula (3).

$$
m=N / n
$$

where $N$ represents the maximum value of the fuzzy subset; $n$ represents the maximum value of the continuous domain.

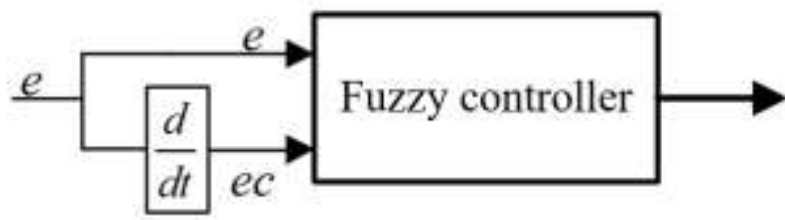

Fig. 4: Two-dimensional fuzzy controller model

\subsection{Fuzzy rules and membership functions}

The design of fuzzy rules is the key to designing fuzzy controllers. It is generally composed of seven words, namely \{negative large, negative medium, negative small, zero, small, medium, and positive\}. It can also be expressed in English as $\{N B, N M, N S, O, P S, P M, P B\}$ [6]. For the fuzzy rule base respectively for $k p, k i, k d$, the relationship between the input function and the membership function of the output variable is shown in Fig. 5 to Fig. 8. 


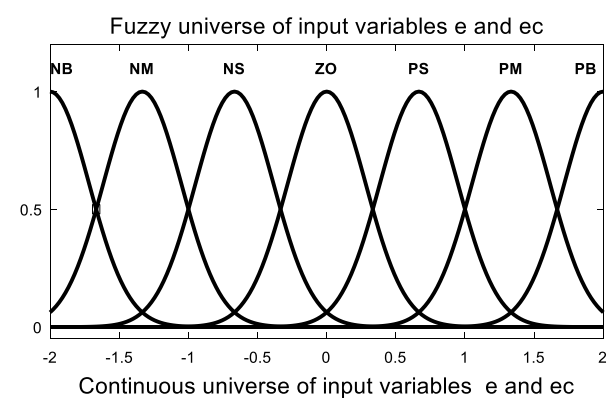

Fig. 5: The input function membership function

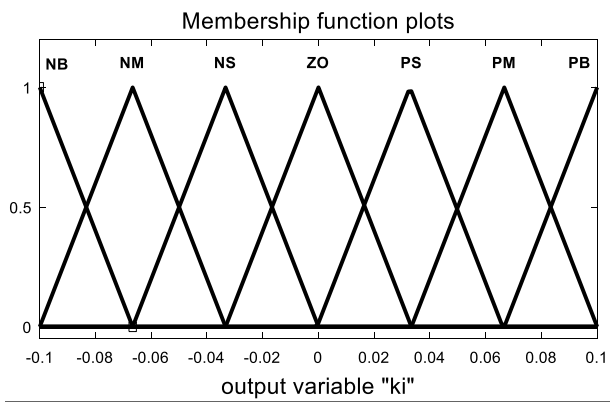

Fig. 7: The ki membership function

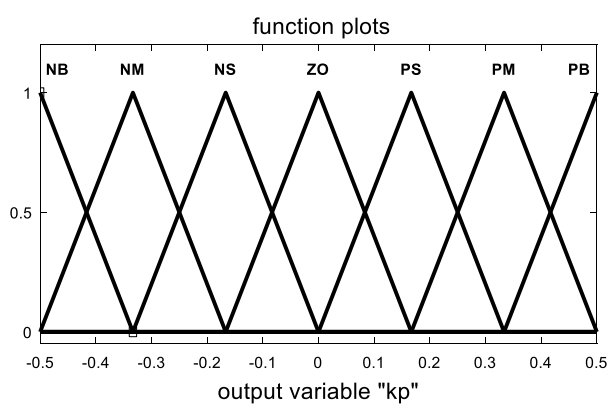

Fig. 6: The $k p$ membership function

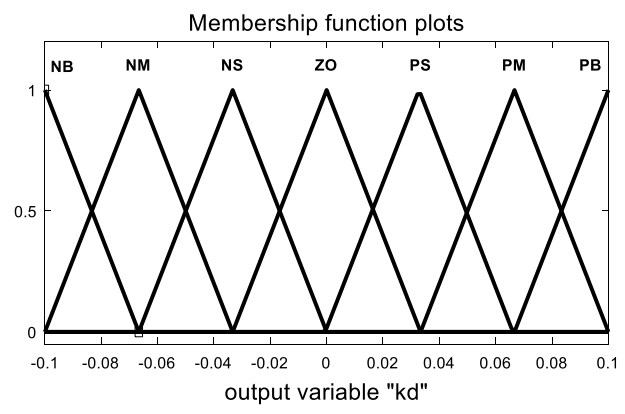

Fig. 8: The $k d$ membership function

\subsection{Building a simulation model}

The fuzzy controller and the membership relationship are set by the fuzzy controller, and the control model is established and simulated by simulink. The simulation model is shown in Figure 9.

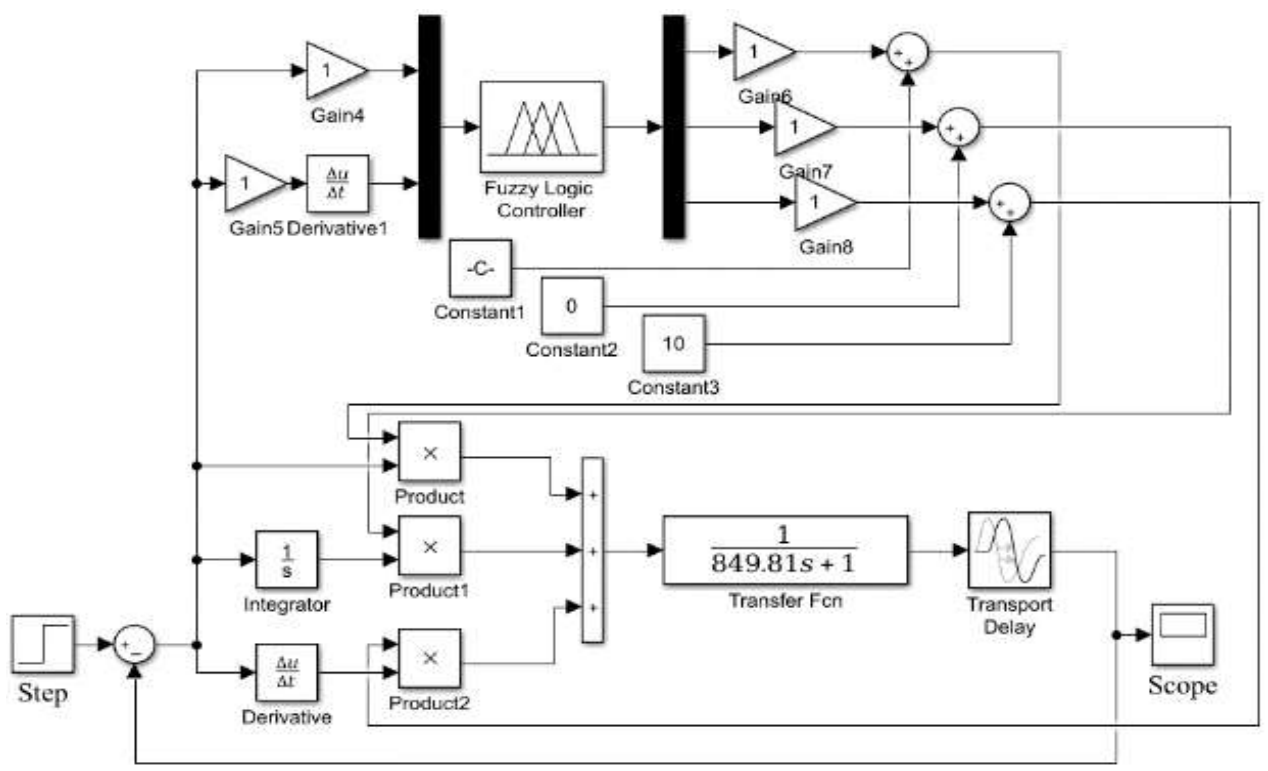

Fig. 9: The fuzzy control simulation model

\section{Particle Swarm PID}

\subsection{Particle swarm optimization}

Particle Swarm Optimization (PSO) is a population-based stochastic optimization technique. Compared with other population-based evolutionary algorithms, they are initialized as a set of random solutions, and the optimal solution is searched by iteration. The difference is that evolutionary computing follows the principle of survival of the fittest, while PSO simulates society [7]. Each possible solution is expressed as a particle in the group, each particle having its own position vector and velocity vector, and a fitness determined by the objective function. All particles fly at a certain speed in the search space, looking for the global optimal value by following the current searched optimal value. The particle swarm optimization algorithm is initialized into a group of random particles, and the optimal solution is found by iteration. In each iteration, the particle updates itself by tracking two extreme values. First, the particle itself finds the optimal solution 
at the current moment. This solution is called The individual optimal value; the second is the optimal solution found by the whole population to the current time, which is the global optimal value, and the particles are continuously updated by the above two extreme values to generate a new generation group [8].

According to Kennedy and Eberhart's particle swarm evolution rules [9]:

$$
\begin{gathered}
v_{i s}(t+1)=\omega v_{i s}(t)+c_{1} r_{1 s}(t)\left(p_{i s}(t)-x_{i s}(t)\right)+c_{2} r_{2 s}(t)\left(p_{g s}(t)-x_{i s}(t)\right) \\
x_{i s}(t+1)=x_{i s}(t)+v_{i s}(t+1)
\end{gathered}
$$

In the formula (1), $v_{i s}$ denotes that the velocity vector $x_{i s}$ of the particle represents the position coordinate of the particle; $p_{i s}$ represents the optimal position searched for the position of the i-th particle so far; $p_{g s}$ represents the optimal position searched for the entire particle group; $\omega$ is a non-negative number, called a dynamic constant, used to control the influence of the previous speed [10]. The local search ability can be adjusted by changing the size of $\omega ; c_{1}, c_{2}$ is called a learning factor; $r_{1}, r_{2}$ is a pseudo-random number independent of each other, obeying the uniform distribution on $[0,1]$.

The algorithm flow of PSO is shown in Figure 10.

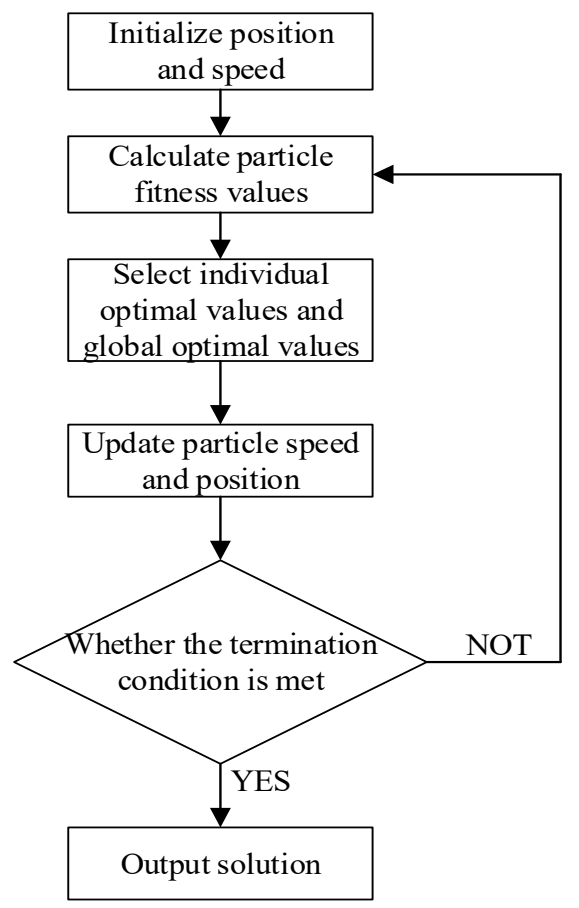

Fig. 10: The optimization Process

\subsection{Particle swarm optimization PID simulation}

According to the steps of particle swarm optimization, the program of particle swarm optimization is first written; secondly, the model of the plant factory is linked with the particle swarm optimization program in the first step; finally, the simulink simulation model of the plant factory needs to be established, as shown in Fig. 11.

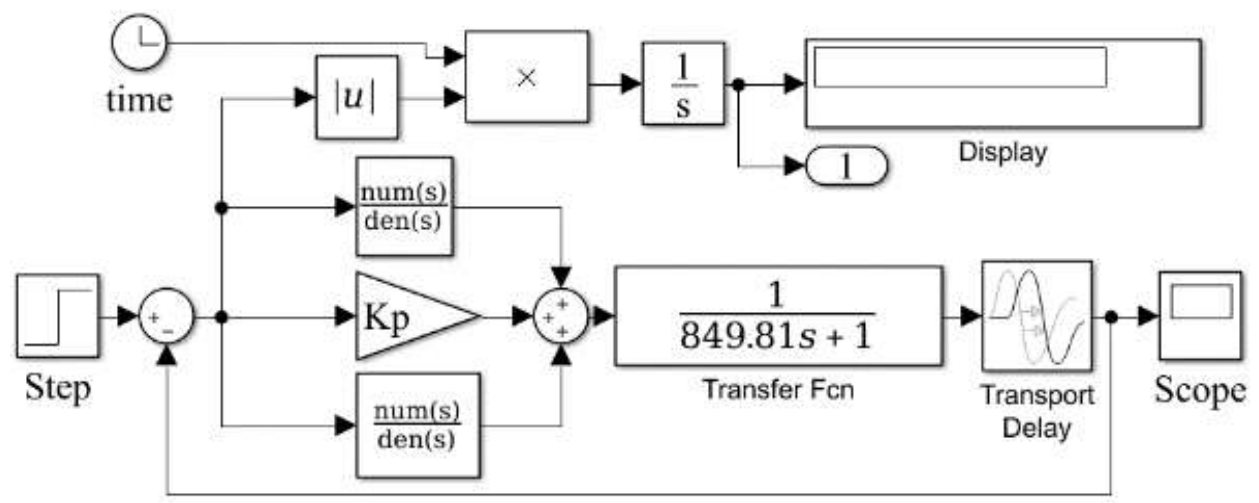

Fig. 11: The PSO PID Simulation Model caption 
The parameters in this paper are set as: particle $M=5$, dimension $\operatorname{dim}=3$, learning factor $c 1=2, c 2=2$, inertia factor $\mathrm{w}=0.6$. After 20 iterations, the optimization results of the quantization factor are shown in Figure 12, and the optimal individual fitness values are shown in Figure 13. According to the optimized curve, $k p=20.58, k i=0.15, k d=20.11$ can be obtained.

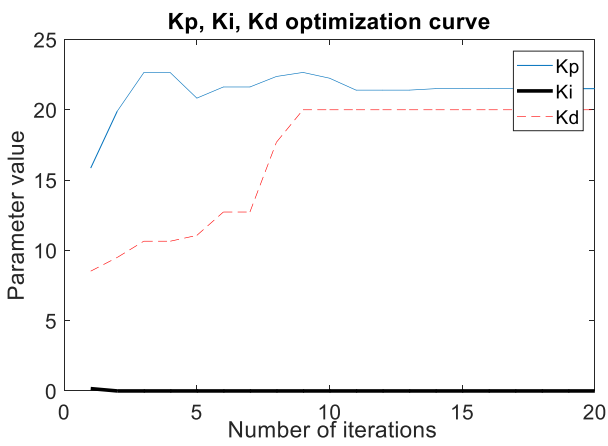

Fig. 12: The quantization factor quantization curve

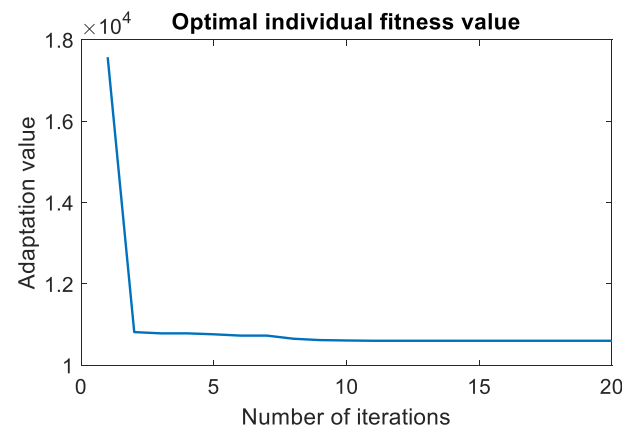

Fig. 13: The optimal individual fitness value curve

\section{Conclusion}

According to the established simulink model and the step response results obtained by simulation, the results show that, as shown in Figure 14, the PID based on particle swarm optimization (PSO) algorithm has strong adaptability and robustness. Sexuality has certain reference significance for the temperature control of plant growth cabinets.

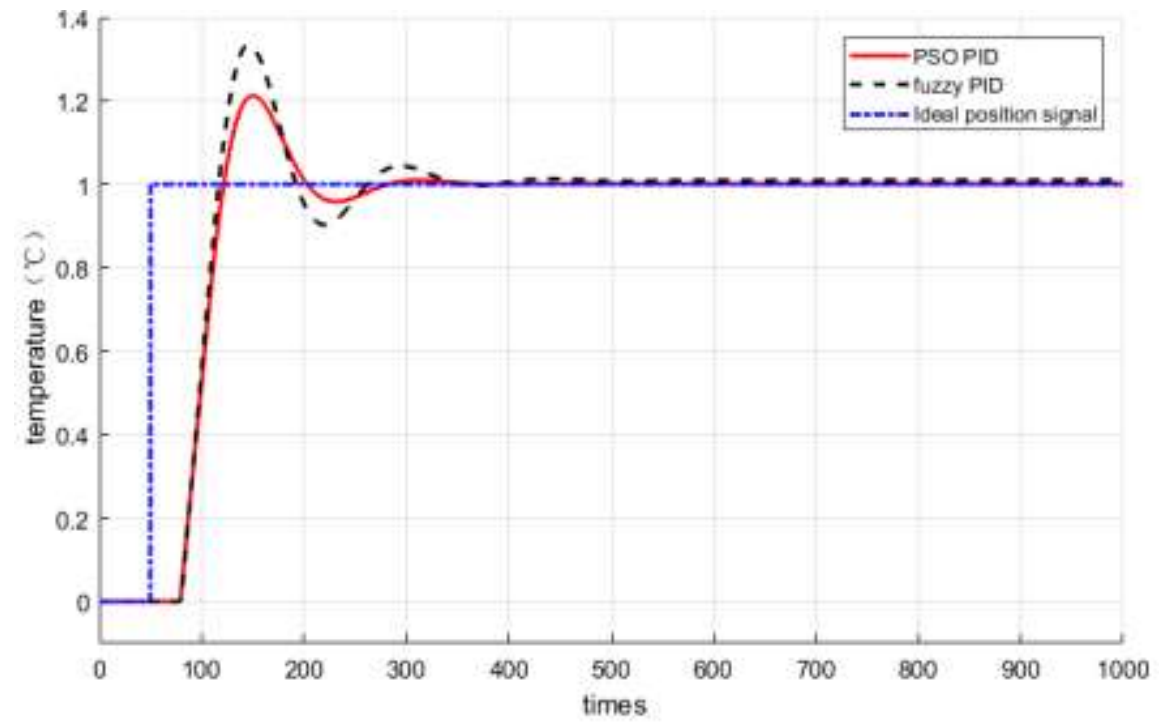

Fig. 14: The simulation result graph

\section{Acknowledgements}

This work was supported by the National Key Research and Development Program (2017YFB0403904) and the Tianjin University of Technology and Education Graduate Innovation Fund Project (YC19-19). 


\section{References}

[1] Kozai T. Plant factory in Japan — current situation and perspectives[J]. Chronica Horticulturae, 2013, 53(2): 8-11.

[2] Hamilton J D. China and the Global Energy Crisis: Development and Prospects for China's Oil and Natural Gas[J]. Energy Journal, 2008, 29(02): 185-186.

[3] Ikeda, Hideo. Plant Factory [J]. Transactions on Sensors and Micro machines. 2011, 131 (6): 200-205.

[4] Scott S A, Davey M P, Dennis J S, et al.Biodiesel from algae:challenges and prospects[J].Current Opinion in Biotechnology, 2010, 21:277-286.

[5] Cooper, W.J, López, et al. Development of the nutrient film technique for renovation of wastewater for nonpotable reuse[J]. Cooper W J, 1983.

[6] Zadeh L A. Fuzzy Sets[J]. Information and Control, 1965, 65(8):338-353.

[7] Mamdani E.H.A Linguistic Self-Organizing Process Controller [J]. Automatica, 1979, 79(15):15-30.

[8] Timothy Ganesan,Pandian Vasant,Irraivan Elamvazuthy. A hybrid PSO approach for solving non-convex optimization problems[J]. Archives of Control Sciences,2012,22(1).

[9] Cvetko J. Andreeski,Pandian Vasant. Simplified Azbel Model for Fitting Mortality Tables[J]. IFAC Proceedings Volumes,2008,41(2).

[10] Schneider, N., M. Gerber, Correlation between viscosity, temperature and total solid content of algal biomass[J]. Bioresource technology, 2014. 170: 293-302. 